D) Check for updates

Cite this: RSC Adv., 2019, 9, 12813

DOl: $10.1039 / c 9 r a 90029 b$

www.rsc.org/advances

\title{
Retraction: First-principle investigations of structural, electronic, magnetic and optical properties of bulk $\mathrm{BiVO}_{3}$
}

\author{
Andrew Shore
}

Retraction of 'First-principle investigations of structural, electronic, magnetic and optical properties of bulk $\mathrm{BiVO}_{3}^{\prime}$ by Biao Liu et al., RSC Adv., 2016, 6, 92473-92478.

The Royal Society of Chemistry hereby wholly retracts this RSC Advances article as recent recalculations on the data have shown that the results in the paper are unreliable.

The original calculation of the DOS (density of states) of $\mathrm{BiVO}_{3}$ in the C-AFM structure is inaccurate. The authors have also recalculated the energies of the FM, A-AFM, C-AFM and G-AFM structures and found that, although the C-AFM phase is the lowest energy structure, the energy difference values of the FM, A-AFM and G-AFM relative to C-AFM are inaccurate in the paper. In addition, the authors found that the structural data for $\mathrm{BiVO}_{3}$ with $P m n a$ and $C 2 / C$ structures in Table 1 is incorrect.

The recalculated results mean that the conclusions of the published paper are now unreliable; therefore, the article is being retracted to protect the accuracy and integrity of the scientific record.

The corresponding author, Meng-Qiu Cai, agreed to retract the article, but did not respond to any correspondence regarding the wording of the retraction notice. The co-authors, Biao Liu, Li-Juan Wu, Yu-Qing Zhao and Lin-Zhi Wang, were contacted but did not respond.

Signed: Andrew Shore, Executive Editor, RSC Advances

Date: $10^{\text {th }}$ April 2019 